\title{
The Kilo-Degree Survey
}

\author{
Jelte T. A. de Jong • Gijs A. Verdoes Kleijn • \\ Konrad H. Kuijken • Edwin A. Valentijn • \\ KiDS and Astro-WISE Consortiums
}

Received: 30 January 2012 / Accepted: 6 June 2012 / Published online: 1 August 2012

(C) The Author(s) 2012. This article is published with open access at Springerlink.com

\begin{abstract}
The Kilo Degree Survey (KiDS) is a 1500 square degree optical imaging survey with the recently commissioned OmegaCAM wide-field imager on the VLT Survey Telescope (VST). A suite of data products will be delivered to the European Southern Observatory (ESO) and the community by the KiDS survey team. Spread over Europe, the KiDS team uses AstroWISE as its main tool to collaborate efficiently and pool hardware resources. In Astro-WISE the team shares, calibrates and archives all survey data. The data-centric architectural design realizes a dynamic 'live archive' in which new KiDS survey products of improved quality can be shared with the team and eventually the full astronomical community in a flexible and controllable manner.
\end{abstract}

Keywords Wide-field imaging • Survey system • VLT/VST • Weak gravitational lensing $\cdot$ Photometric redshifts

\section{Introduction}

One of the radical advances that optical astronomy has seen in recent years is the advent of wide-field CCD-based surveys. Key ingredients for these surveys

\footnotetext{
J. T. A. de Jong · K. H. Kuijken

Leiden Observatory, Leiden University, P.O. Box 9513,

2300 RA Leiden, The Netherlands

e-mail: jelte@strw.leidenuniv.nl

G. A. Verdoes Kleijn $(\varangle) \cdot$ E. A. Valentijn

Kapteyn Astronomical Institute, University of Groningen,

P.O. Box 800, 9700 AV Groningen, The Netherlands

e-mail: verdoes@astro.rug.nl
} 
are the availability of instruments with sufficiently large arrays of high quality CCD's, as well as information systems with sufficient computing and data storage capabilities to process the huge data flows. The scientific importance of such surveys, particularly when freely available to researchers, is clearly demonstrated by the impact that surveys such as 2MASS [29] and the Sloan Digital Sky Survey (SDSS) [1], have had in several fields in astronomy.

Due to their telescopes being located in the Northern hemisphere, both SDSS and the currently on-going PanSTARRS survey ${ }^{1}$ mainly survey the Northern sky. So far, a similar large scale survey has not been performed from the South and no dedicated optical survey telescope were operational until recently. For European astronomy, however, the Southern hemisphere is especially important, due to the presence of ESO's Very Large Telescope (VLT) and its large array of instruments. This is now remedied with the arrival of ESO's own two dedicated survey telescopes: VISTA in the (near-)infrared and the VLT Survey Telescope (VST) in the optical. Both have become operational during the past two years. The lion's share of the observing time on both survey telescopes will be invested in a set of 'public surveys'. In terms of observing time, the largest of the optical surveys is the Kilo-Degree Survey (KiDS), which is imaging 1500 square degrees in four filters $(u, g, r, i)$ over a period of 3-4 years. Combined with one of the VISTA surveys, VIKING, ${ }^{2}$ which is observing the same area in ZYJHK, this will provide a sensitive, 9band multi-colour survey.

Specifically for the handling of surveys from the VST the Astro-WISE system [31] has been designed. It allows processing, quality control and public archiving of surveys using a distributed architecture. The KiDS survey team uses Astro-WISE as its main tool to handle the survey data, facilitate scientific analysis of the data, and to create public data products. Astro-WISE is Virtual Observatory enabled, facilitating straightforward porting of data, at any processing stage, to external analysis pipelines within the KiDS consortium (e.g. THELI/Lensfit $[9,23]$ for weak lensing applications). The KiDS survey team, that is spread over different countries, can work as a single virtual team making intensive use of web-based collaborative interfaces. This paper will discuss both the observational set-up of the KiDS survey and its primary scientific goals, as well as how the Astro-WISE system will facilitate achieving these goals.

\section{The Kilo-Degree Survey}

\subsection{Instrumental set-up}

The VST is located at Paranal Observatory in Chile and operated by ESO. Regular observations with the system commenced on 15 October 2011. With

\footnotetext{
${ }^{1} \mathrm{http}: / / \mathrm{ps} 1 \mathrm{sc}$. org

${ }^{2}$ http://www.eso.org/sci/observing/policies/PublicSurveys/sciencePublicSurveys.html
} 

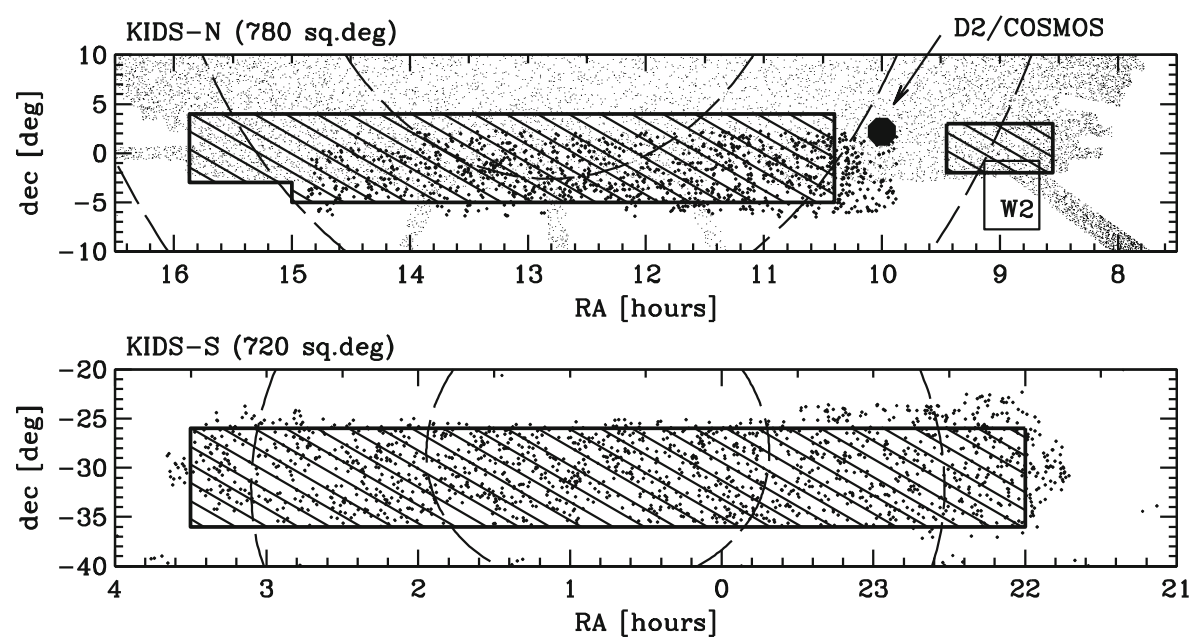

Fig. 1 Lay-out of the KIDS-North (top) and KIDS-South (bottom) fields, shown by the hatched areas. Also shown are the areas where $2 \mathrm{dF}$ spectra are available, indicated by the large dots, and the area covered by DR7 of the SDSS survey, indicated by the small dots. The CFHTLS-W2 field and the DS/COSMOS deep field are overplotted on the top panel

a primary mirror of 2.6-m diameter it is currently the largest telescope in the world specifically designed for optical wide-field surveys. The sole instrument of the VST is OmegaCAM [15], a 268 Megapixel wide-field camera that provides a $1^{\circ} \times 1^{\circ}$ field-of-view. The focal plane array is built up from $322048 \times$ 4096 pixel CCDs, resulting in $16 \mathrm{k} \times 16 \mathrm{k}$ pixels with a pixel scale of 0.214 arcseconds/pixel. The optics of the telescope and camera were designed to produce a very uniform point-spread-function over the full field-of-view, both in terms of shape and size. For a more detailed description we refer the reader to the OmegaCAM paper in this issue [33].

\subsection{Observational survey set-up}

KiDS will cover 1500 square degrees, some $7 \%$ of the extragalactic sky. It consists of two patches, ensuring that observations can take place year-round. The Northern patch lies on the celestial equator, while the Southern patch straddles the South Galactic Pole; see Fig. 1 and Table 1 for the detailed lay-out.

Table 1 KiDS fields

\begin{tabular}{llll}
\hline Field & RA range & Dec range & Area \\
\hline KiDS-S & $22^{h} 00^{m}-3^{h} 30^{m}$ & $-36^{\circ}$ to $-26^{\circ}$ & 720 sq.deg \\
KiDS-N & $10^{h} 24^{m}-15^{h} 00^{m}$ & $-5^{\circ}$ to $+4^{\circ}$ & 712 sq.deg \\
& $15^{h} 00^{m}-15^{h} 52^{m}$ & $-3^{\circ}$ to $+4^{\circ}$ & \\
KiDS-N-W2 & $8^{h} 30^{m}-9^{h} 30^{m}$ & $-2^{\circ}$ to $+3^{\circ}$ & 68 sq.deg \\
KiDS-N-D2 & $9^{h} 58^{m}-10^{h} 02^{m}$ & $+1^{\circ}$ to $+3^{\circ}$ & 2 sq.deg \\
\hline
\end{tabular}


Together the two patches cover a range of galactic latitudes from $40^{\circ}$ to $90^{\circ}$, and the $10^{\circ}$ width of the strips ensures that the full 3D structure of the universe is sampled well. These specific areas were chosen because they have been the target of massive spectroscopic galaxy surveys already: the $2 \mathrm{dF}$ redshift survey [6] covers almost the same area, and KiDS-N overlaps with the SDSS spectroscopic and imaging survey as well. This means that several 100,000 galaxy spectra and redshifts are already known in these fields, and hence that the cosmological foreground mass distribution in these fields is well mapped out. Extinction in the fields is low. The exposure times for KiDS and VIKING have been chosen to yield a median galaxy redshift of 0.8 , so that the evolution of the galaxy population and matter distribution over the last $\sim$ half of the age of the universe can be studied. They are also well-matched to the natural exposure times for efficient VST and VISTA operations, and balanced over the astro-climate conditions on Paranal (seeing and moon phase) so that all bands can be observed at the same average rate. This strategy makes optimal use of the fact that all observations are queue-scheduled, making it possible to use the best seeing time for deep $r$-band exposures, for example, and the worst seeing for $u$. All exposure times and observing constraints are listed in Table 2, where seeing refers to the full-width-half-maximum (FWHM) of the point-spread-function (PSF) measured on the images.

Since the OmegaCAM CCD mosaic consists of 32 individual CCDs, it is not contiguous but contains gaps. To avoid holes in the KiDS images, observations will use 5 dithered observations per field in $g, r$ and $i$ and 4 in $u$. The dithers form a staircase pattern with dither steps of $25^{\prime \prime}$ in $\mathrm{X}$ and $85^{\prime \prime}$ in Y. These offsets bridge the inter-CCD gaps of OmegaCAM. The survey tiling is derived using a tiling strategy that can tile the full sky efficiently for the OmegaCAM instrument. Neighboring tiles have an overlap in RA of 5\% and in DEC of $10 \%$. This will allow us to derive the photometric and astrometric accuracies required for the most stringent science cases: internal astrometric error $<0.03^{\prime \prime} \mathrm{rms}$ and $1 \%$ photometric errors. The Atmospheric Dispersion Corrector (ADC) of OmegaCAM could be used for all KiDS bands except $u$. However, KiDS does not make use of the ADC to avoid the small losses in sensitivity. Instead, the dispersion is limited by constraining the maximum airmass (see Table 2). Particularly for $r$ this is important since this band will be used for weak lensing analyses and therefore requires a well-behaved PSF. By constraining the maximum airmass in $r$ to 1.3, the spectral dispersion will be $<0.2^{\prime \prime}$.

Table 2 KiDS exposure times and observational constraints

\begin{tabular}{lllllll}
\hline Filter & $\begin{array}{l}\text { Exposure } \\
\text { time }(\mathrm{s})\end{array}$ & $\begin{array}{l}\text { Mag limit } \\
\left(\mathrm{AB} 5 \sigma 2^{\prime \prime}\right)\end{array}$ & $\begin{array}{l}\text { PSF FWHM } \\
(\operatorname{arcsec})\end{array}$ & $\begin{array}{l}\text { Moon } \\
\text { phase }\end{array}$ & $\begin{array}{l}\text { ADC } \\
\text { used }\end{array}$ & Airmass \\
\hline$u$ & 900 & 24.8 & $0.9-1.1$ & Dark & no & 1.2 \\
$g$ & 900 & 25.4 & $0.7-0.9$ & Dark & no & 1.6 \\
$r$ & 1800 & 25.2 & $<0.8$ & Dark & no & 1.3 \\
$i$ & 1080 & 24.2 & $<1.1$ & Any & no & 2.0 \\
\hline
\end{tabular}


After completion of the main survey of 1500 square degrees, the whole survey area will be imaged once more in $g$-band. The observational set-up of this repeat pass is the same as for the main survey observations in $g$, with the additional requirement that it should provide at least a 2 year baseline over the whole survey area. With the average $g$-band seeing of $0.8^{\prime \prime}$ this will allow for proper motion measurements with accuracies of $40 \mathrm{mas} \mathrm{yr}^{-1}$ and better for sources detected at signal-to-noise of 10 [16].

\subsection{Scientific goals}

The central science case for KiDS and VIKING is mapping the matter distribution in the universe through weak gravitational lensing and photometric redshift measurements. However, the enormous data set that KiDS will deliver, will have many more possible applications. The main research topics that the KiDS team members will explore are outlined below.

\subsubsection{Dark energy}

Dark energy manifests itself in the expansion history of the universe, as a repulsive term that appears to behave like Einstein's cosmological constant. Understanding its properties more accurately is one of the central quests of cosmology of recent years (e.g. [35]). With KiDS we intend to push this question as far as possible, while recognising that the limiting factor may well be systematic effects rather than raw statistical power. Measurements done with KiDS will therefore also serve as a learning curve for future (space-based) experiments.

The use of weak gravitational lensing as a cosmological probe is nicely summarized in [22] and [26]. Essentially, its power relies on two facts: gravitational lensing is a very geometric phenomenon, and it is sensitive to mass inhomogeneities along the lines of sight.This makes it a good probe of the growth of structure with time (redshift), as well as being a purely geometric distance measure. As it happens, the distance-redshift relation and the speed with which overdensities grow with cosmic time are the two most fundamental measures of the energy content of the universe: both depend directly on the rate at which the universe expands. Making such a measurement, for which weak lensing is an excellent method, is therefore of great interest. These lensing measurements are not easy, as they require systematics better than $1 \%$ accuracy, and photometric redshifts unbiased at a similar level. However, with KiDS we have put ourselves in the optimal position to attempt this, by ensuring the best image quality in our instrument, by choosing a survey depth and area appropriately, and having a wide wavelength coverage that will make the photometric redshifts as free of error as is possible with wideband photometry (see Fig. 2). As noted above, the expansion history can be deduced from lensing tomography in several ways, and requiring consistency is a powerful check-as well as, further down the line, an interesting test of Einstein gravity theory [13]. 

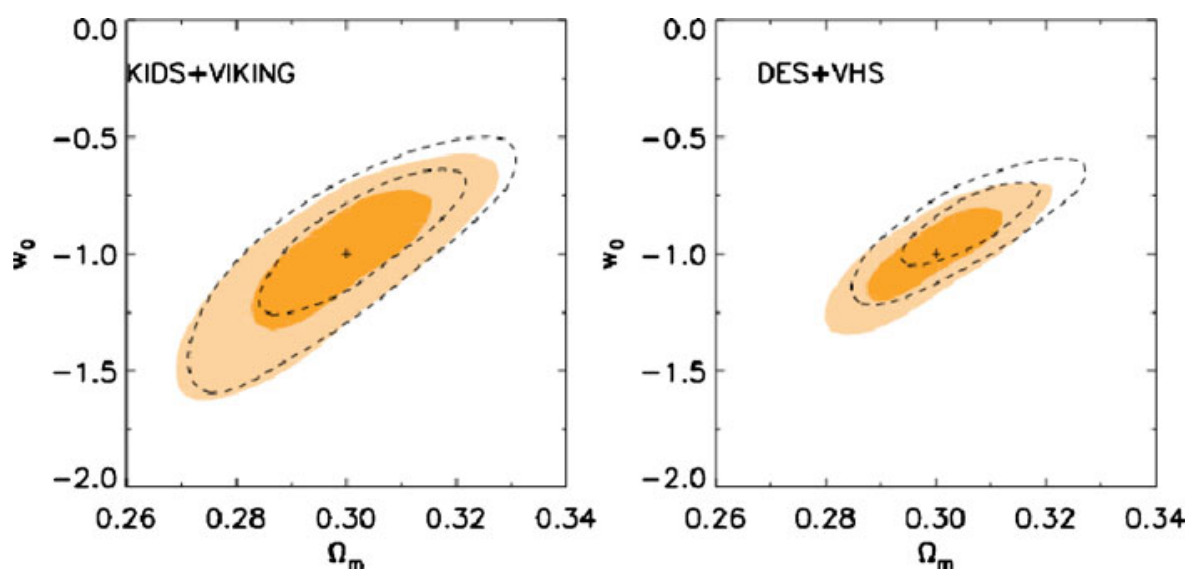

Fig. 2 Comparison of the formal statistical power and sensitivity to systematic errors in the photometric redshifts for the KiDS/VIKING and DES/VHS surveys in cosmological parameter estimation (here the matter density $\Omega_{m}$ and the dark energy equation of state $w_{0}$ ), based on simulated photometry for each of the surveys (Szomoru et al., 2012, private communication). The '+' represents the input truth. The coloured contours assume perfect redshift information, while the dashed contours show the effect of redshift errors. Flat geometry was assumed here, but otherwise no external information were included. Once external information is folded in, the constraints tighten and systematic effects become even more significant, demonstrating the greater robustness of the KiDS survey to this type of systematic error

An independent way to study the expansion history of the universe is by measuring the baryon acoustic oscillations (BAO). BAO is the clustering of baryons at a fixed co-moving length scale, set by the sound horizon at the time that the universe recombined and photons decoupled from baryonic matter. This scale length, which has been measured accurately in the cosmic microwave background [30], is therefore a standard ruler, whose angular size on the sky provides a direct measurement of the angular diameter-redshift relation and hence of the expansion history. Using photometric redshifts from KiDS we can make an independent measurement of the BAO scale. Comparison of the results with recent and ongoing spectroscopic BAO surveys such as WiggleZ [8] and BOSS [28] provides a potent test of systematics. Simulations of photometric redshift measurements using full 9-band coverage, ugrizYJHK as will be provided by KiDS and VIKING, have shown that the accuracy needed for detection of the BAO signal $(0.03 \times(1+z)$ rms photometric redshift error) can be reached. Tests of the detectability of the BAO with particle and Monte-Carlo simulations, provided by Peter Schuecker, have shown that imaging surveys of the size and sensitivity of KiDS can yield values of $w$ with $\sim 5 \%$ accuracy.

\subsubsection{Structure of galaxy halos}

Simulations of structure formation provide detailed information about the shape of dark matter halos on large scales. However, at small scales such as the 
inner parts of galaxy halos, complex physics that these simulations can not represent realistically (e.g. star formation, cooling, feedback etc.) starts to play an important role (e.g. [32]). The relation between light (baryons) and mass (dark matter) is crucial for our understanding of the influence of the dark matter on galaxy formation, and vice-versa. Galaxy-galaxy lensing (GGL) provides a unique way to study this relation between galaxies and their dark halos.

The gravitational lensing effect of foreground galaxies on the images of background galaxies is very weak, and can only be measured statistically. This is done by stacking large numbers of foreground galaxies and measuring the net image distortion of the background galaxies. On small scales (3-30 arcsec) the signal is dominated by the profile of the foreground galaxies' inner dark matter halos, at radii of 10-100 s of kpc. At scales of several arcminutes GGL probes the galaxy-mass correlation and the bias parameter, while at even larger scales the distribution of the foreground galaxies in their parent group halos dominates. GGL can therefore be used to probe halos over a large range of scales and help to test the universality of the dark halo profile.

The strength of KiDS for GGL is again twofold. The shear size and thus enormous numbers of available galaxies, makes it possible to split the foreground galaxies in bins and study different galaxy types separately. The accurate photometric redshifts also allow splitting up the samples in redshift bins, thus enabling the redshift dependence to be analyzed. Furthermore, the fact that KiDS targets areas where wide-field redshift surveys have already been carried out, means that the foreground large scale structure is known, enabling the measurement of the galaxy-mass correlation for galaxy groups, clusters, and even filaments. Compared to earlier GGL studies with, for example, SDSS [19] or CFHTLS [25], the image quality and sensitivity of KiDS will provide many more foreground-background pairs, more accurate shape measurements, and the ability to probe the galaxy population at higher redshifts.

\subsubsection{Evolution of galaxies and clusters}

Within the current cosmological paradigm of Cold Dark Matter (CDM), structure formation is hierarchical and the profiles of CDM halos are universal, i.e. the same at all scales. Several of the ramifications of this picture have so far eluded rigorous observational testing. For example, various observational constraints on the influence of galaxy mergers on the evolution of the galaxy population at redshifts higher than $\sim 0.5$ differ up to an order of magnitude (see e.g. [17]). The observational studies targeted small numbers of galaxies $(<1000)$ at high spatial resolution (e.g., [18]) or small areas ( $<1$ square degree, e.g. [7]). Also, galaxy clusters are probes of the highest mass peaks in the universe, but at redshifts of $z>1$ the number of known galaxy clusters is yet too small to constrain cosmological models.

KiDS can play a major role in this field. The sensitivity of the KiDS photometry will result in the detection of an estimated $10^{8}$ galaxies. This galaxy sample will have a median redshift of $z=0.8$, with $\sim 20 \%$ having $1<z<1.5$. Based on this sample the evolution of the galaxy luminosity function, the 
build-up of stellar mass and the assembly of early-type stellar systems can be traced back to unprecedented look-back times.

Cluster finding will be possible directly from the multi-colour KiDS catalogues. In total we expect KiDS to provide $1-2 \times 10^{4}$ clusters, and with the red sequence detectable out to $z \sim 1.4$ approximately $5 \%$ of these will be located at redshifts beyond 1 . This will be a very important sample to further constrain cosmological parameters, provided that the relation between cluster richness and cluster mass can be calibrated. This calibration is possible since the weak lensing measurements that will be done as part of KiDS will probe the cluster mass distribution, demonstrating the pivotal advantage of combining high image quality with uniform multi-band photometry.

A different perspective of galaxy evolution will be provided by virtue of the fact that that KiDS-S overlaps two nearby superclusters (Pisces-Cetus and Fornax-Eridanus). Thus, the relation between galaxy properties (e.g. star formation rate) and environment, can be studied all the way from cluster cores to the infall regions, and to the filaments that connect clusters in the cosmic web.

\subsubsection{Stellar streams and the galactic halo}

Detailed studies of the stellar halo of the Milky Way require photometry of faint stars over large areas of sky. The SDSS, although primarily aimed at cosmology and high-redshift science, has proved a milestone in Milky Way science as well, unveiling many stellar streams and unknown faint dwarf Spheroidal galaxies [4, 5]. While KiDS will image a smaller area than SDSS, it is deeper and thus will provide a view on more distant parts of the halo. But more importantly, SDSS only covered the Northern sky, leaving the Southern hemisphere as uncharted territory. Particularly in the KiDS-S area, new discoveries are bound to be made in the direct vicinity of our own Galaxy.

\subsubsection{Proper motions}

Proper motions with accuracies of $\sim 0.04^{\prime \prime} \mathrm{yr}^{-1}$ will be available in the KiDS area, owing to the planned g-band repeat pass that will provide a 2-year baseline. Several applications are possible, among others the detection and study of high proper-motion white dwarfs. "Ultracool" white dwarfs $(<4000 \mathrm{~K})$, relics from the earliest epochs of star formation, are among the oldest objects in the Galaxy and can be used to trace the very early star formation history of our Galaxy. Due to its multicolour photometry combined with proper motion information, KiDS will be able to increase the sample of known, ultracool white dwarfs $[12,14]$ significantly.

\subsection{Survey calibration and data products}

Being a Public Survey, all KiDS data will be made publicly available. The KiDS catalogue will contain some 100,000 sources per square degree (150 million 
sources over the full survey area), and for each square degree there will be $10 \mathrm{~GB}$ of final image data, $15 \mathrm{~TB}$ for the whole survey. These data will be of no use if they are not uniformly and carefully calibrated and made available in an easily accessible archive.

The astrometric calibration is done per tile (stack of dithers) using the gnomonic tangential projection and using the 2MASS Point Source Catalog (2MASS PSC, [29]) as survey astrometric reference. The first step is a local astrometric solution. An astrometric model for a single chip is constrained by the star positions from a single exposure. The local solution is the input for global astrometry. The global solution uses a global model of the focal plane that allows for variations over the dither positions that make up the tile (e.g., due to telescope flexure and field rotation tracking residuals). The observational constraint comes from the internal positional residuals from dither overlaps and external residuals with the 2MASS PSC. First results for local astrometry indicate $0.1^{\prime \prime}-0.15^{\prime \prime} \mathrm{rms}$ in relative astrometry for KiDS. Global astrometry is expected to yield $\sim 0.05^{\prime \prime}$ rms.

The photomeric calibration will be done over the survey as a whole in Sloan photometric system using AB magnitude system. The Calibration Plan of the survey telescope includes nightly zeropoints on SA standard fields in $u, g, r$ and $i$. A dedicated OmegaCAM calibrational observing program is obtaining Secondary Standards in the SA fields covering the full OmegaCAM FoV. The program is expected to run until the last quarter of 2012. Each night $\sim 3$ observations are taken of a fixed polar standard field near the southern equatorial pole for atmospheric monitoring using a composite filter with $u, g, r$ and $i$ quadrants. Daily domeflats are taken in all bands to measure the system throughput (telescope plus camera). Commissioning results indicate that the on-sky observations yield photometric scales to $<1 \%$ accuracy and the in dome observations an $<0.5 \%$ accuracy. The overlaps between KiDS science observations constrain the relative photometric calibration over the full survey. For the absolute photometric calibration the standard field observations are used. The fellow ATLAS survey ${ }^{3}$ using OmegaCAM on the VST has full overlap with the KiDS-S area and $\sim 25 \%$ overlap with the KiDS-N area in $u, g, r$ and $i$. Tying the KiDS survey to these ATLAS areas plus the standard fields shall prevent calibration "creep" from the tile-to-tile photometric calibration. McFarland et al. [21] give a detailed description of the Astro-WISE calibration pipeline. The OmegaCAM and VST calibration is discussed in detail in [33]. They also discuss the instrument photometric characterization, including illumination variations.

Basic data products will be made public, both through ESO and through the Astro-WISE database, within a year after any part of the survey area has been observed in all filters. This set of basic data products includes the following:

- astrometrically and photometrically calibrated coadded and regridded images with weight maps;

${ }^{3}$ http://astro.dur.ac.uk/Cosmology/vstatlas/ 
- calibration images: twilight flats, dome flats, biases, fringe maps, etc.;

- single-band source catalogues;

- multi-color (i.e. combined single-band) catalogues.

In addition, and on a longer time-scale, we intend to provide more refined and advanced data products. In the context of the lensing project for KiDS several innovative image processing techniques have been developed, and to the extent possible these will be used to generate high-level data products in the KiDS database. Many of the parameters developed for the SDSS survey will be provided. Furthermore we will look at including:

- Images with "Gaussianized" PSF. Versions of all images convolved with kernels chosen to result in a homogenized, round and Gaussian PSF, to ease comparison with images taken at different times or with different filters or instruments.

- Aperture-matched colour catalogues. Catalogues with colours measured only from the high $\mathrm{S} / \mathrm{N}$ inner regions of sources, for applications that only require flux ratios, rather than total fluxes.

- Unsharp masked images. A wealth of underlying galaxy structure can be obtained from images in which the low frequencies have been removed (dust structures, disks, etc.). We plan to provide images filtered in various ways.

- Morphological parameters. The popular galaxy profile fitting programmes GALFIT [27] and GALPHOT [10] have been implemented in AstroWISE, and will be run on the sources and published in the KiDS database.

The public data products of the VIKING survey will be enhanced and complemented by the KiDS team with combined optical-infrared imaging and catalog data. For a more extensive discussion on how VIKING data products will be ingested and processed (where needed) within Astro-WISE, see the Data Zoo paper in this issue [34].

\section{Internet-based survey collaboration for a geographically distributed team}

The KiDS survey team is an international collaboration with $\sim 35$ team members at institutes spread around Europe and beyond, and has a large variety of scientific interests, as described above. This allows us to perform a thorough quality control of KiDS from many different angles and it ensures a high-quality data product for the ESO community. Where necessary the team will develop new algorithms and complement the public release with specialised products for the most demanding scientific applications, e.g. weak lensing. In this section we focus on the survey handling that is involved in the production of the versatile set of public data products described above. For this task the team uses Astro-WISE: data processing (image calibration, stacking, cataloging), data quality control and data management (on-line archiving and publishing in the Virtual Observatory). By logging on to a single system, 
Astro-WISE, team members can make use of a distributed pool of storage, compute and database resources spread over Europe, and do their survey work, irrespective of where this person is. Day-to-day survey handling is done via webservices. Thus, a web browser with internet connection is all that is required to start doing KiDS survey work.

\subsection{Astro-WISE: a data-centric approach}

This 'whoever, wherever' approach is possible because all aspects of survey handling in Astro-WISE are implemented from a data-centric viewpoint. For example, calibration scientists add information on the time-validity of a calibration item (e.g. a master flatfield image) to this item: the information becomes part of the data item itself. Quality control is handled similarly, since the verdicts of both automatic and human quality assessors become part of the data items themselves. The same is true for data management, because rather than users knowing which data they can reach, in Astro-WISE each data item 'knows' (contains information on) which users it can reach. Processing is implemented as data reaching compute clusters, not as users reaching compute clusters, and all survey data can access all hardware that is pooled by the KiDS team.

Moreover, a survey data item also 'knows' how it was made, and whether it could be made better using new, improved calibrations. This is possible because all processing operations are implemented as actions by data objects acting upon themselves and/or other data objects. Each type of survey product, from raw to final, is represented by a class of data objects, and each survey product is a data object: an informational entity consisting of pixel and/or metadata, where metadata is defined as all non pixel data. Final survey products also carry the information on how they can be created out of intermediate survey objects. This backward chaining procedure is recursively implemented up to the raw data (see Fig. 3 and [24]). Thus, a request by a KiDS team member for a survey product, a target, triggers a backward information flow, in the direction of the raw data. The net effect is a forward work flow description, towards the target, that is then executed. The backward information flow is implemented as queries to database initiated by the requested target itself. The database is queried for survey objects on which the target depends with the right characteristics including validity and quality. Either they exist and are returned or the query is 'backwarded' to the next level of survey objects closer to the raw data.

In conclusion, Astro-WISE takes a data-centric approach to survey handling and control. Attributes of data objects solely determine which calibration data are applied to which science data, which survey products have been qualified and which products should be considered experimental or baseline. Data processing is realized by backward information flows that results in a forward processing flow. See the WISE paper in this special issue [2] for more information on Astro-WISE itself. 


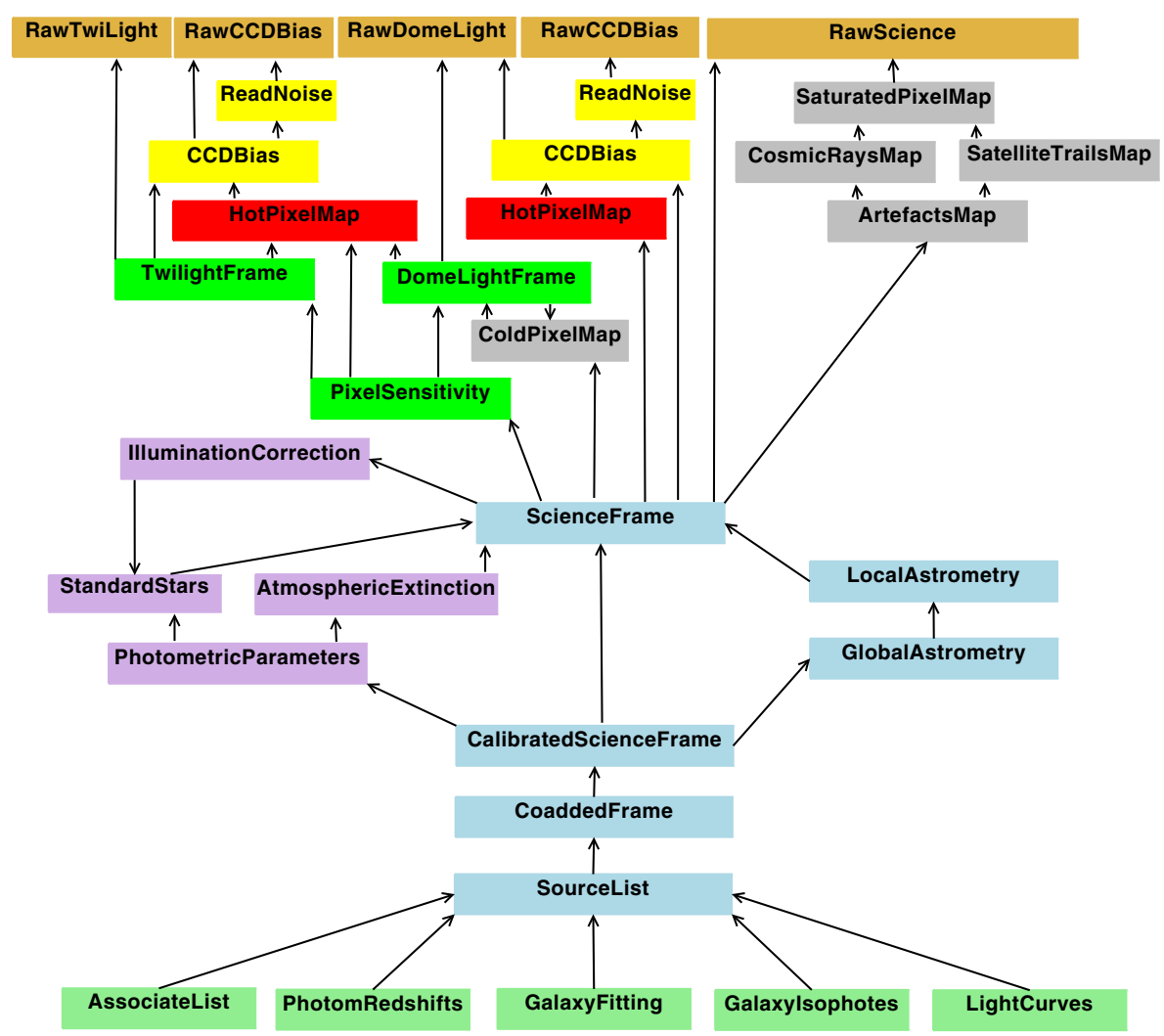

Fig. 3 Overview of classes of data objects. Data objects not only contain the survey products denoted by familiar names in wide-field imaging. They also carry the information how they, as requested target, can be created out of other survey objects, illustrated by the arrows. Underlying is an object model that captures the relationship between requested information and the physics of the atmosphere-to-detector observational system

\subsection{Managing the KiDS survey data}

Handling of KiDS survey data (and any dataset in Astro-WISE) is based on a number of parameters and data attributes that regulate which users can interact with which data objects, and contain information on the quality and validity of data objects. Now follows a detailed description of these parameters, summarized in Table 3.

1. Creator. Each data object is associated with a single Astro-WISE user, its Creator, the user that created/ingested the data object. An Astro-WISE user is a person with an Astro-WISE database account consisting of an id number and name. Each person has only one account and therefore a single identity within the Astro-WISE system. Once created, the creator of a data object cannot be changed. 
Table 3 Set of data-item attributes used for data management, calibration control, quality assessment and control in Astro-WISE

\begin{tabular}{|c|c|c|}
\hline Attribute & Content description & Possible values \\
\hline _creator & $\begin{array}{l}\text { Astro-WISE user that created the } \\
\text { data object }\end{array}$ & Any Astro-WISE user name \\
\hline _project & Project data object belongs to & Any Astro-WISE project name \\
\hline _privileges & $\begin{array}{l}\text { Operational level at which data } \\
\text { object resides }\end{array}$ & $1,2,3,4,5$ \\
\hline is_valid & Validity indicator set by user & 0 (bad), 1 (no verdict), 2 (good) \\
\hline quality_flags & Quality flag set by system & $\begin{array}{l}\text { Any integer (bitwise), } \\
\text { with } 0=\text { good }\end{array}$ \\
\hline timestamp_start & $\begin{array}{l}\text { Start and end of validity range in } \\
\text { time for a calibration object }\end{array}$ & \\
\hline $\begin{array}{l}\text { timestamp_end } \\
\text { creation_date }\end{array}$ & Time of creation of data object & \\
\hline
\end{tabular}

2. Project. A project in Astro-WISE is a group of Astro-WISE users that share a set of data objects. ${ }^{4}$ A project has a project id, a name, a description, project members and optionally an instrument. One or more users can be member of a project, and a user can be member of more than one project. Each data object belongs to one and only one project, which is chosen upon the creation/ingestion of the data entity and can not be changed after that. Some projects have all Astro-WISE users as members (public projects), while other projects have a subset of Astro-WISE users as members (private projects). The 'KIDS' project is a private project and contains all data objects resulting from processing of KiDS survey data.

3. Privileges. Survey data management is facilitated by having pools of data at five different levels named Privileges levels. The term privileges stems from the data-centric viewpoint of Astro-WISE. Each data object has a _privileges attribute that defines its Privileges level. An object has increasing privileges to access users with numerical increase of its Privileges level. Table 4 lists the five levels and which users a data object can reach at each level. The initial privileges of a data object are set by the creator upon the creation/ingestion of the data entity. This can be changed later by creator and project managers, a process called publishing.

4. is_valid. This data attribute is the validity indicator as set by users. It stores the quality assessment performed by a survey team member. Its default value upon creation of a data item is is_valid $=1$, meaning no user assessment has taken place. The team member can change this to is_valid $=0$, meaning bad quality, or to is_valid $=2$, meaning data is qualified as good.

5. quality_flags. This data attribute collects the quality flags as set by the system. Automatically the quality of objects (i.e., survey products) is verified upon creation. If the quality is compromised the quality_flags are set to

\footnotetext{
${ }^{4}$ For an overview of all projects see http://process.astro-wise.org/Projects.
} 


\begin{tabular}{|c|c|c|}
\hline \multirow{6}{*}{$\begin{array}{l}\text { Table } 4 \text { Sharing KiDS data in } \\
\text { Astro-WISE: privilege levels }\end{array}$} & Privileges level & Data is shared with \\
\hline & 1: MYDB & only the creator \\
\hline & 2: PROJECT & $\begin{array}{l}\text { every member of the project to which } \\
\text { the data object belongs }\end{array}$ \\
\hline & 3: ASTRO-WISE & all Astro-WISE users \\
\hline & 4: WORLD & $\begin{array}{l}\text { the world: Astro-WISE users and persons } \\
\text { without an Astro-WISE account } \\
\text { (latter via webservice DBViewer) }\end{array}$ \\
\hline & 5: $\mathrm{VO}$ & $\begin{array}{l}\text { the whole world and data also } \\
\text { available through the Virtual } \\
\text { Observatory webservices }\end{array}$ \\
\hline
\end{tabular}

a value $\neq 0$. It is a bitwise flag. Each type of object (raw science frames, calibrated science frames, astrometric solutions) has its own definition of what each bit means.

6. timestamp_start, timestamp_end. These attributes of a calibration data item define the range in time for which it is applicable. Calibration data get default timestamp ranges upon creation. These can be modified by survey team members. For example, it might be decided that a zeropoint might apply to one or more nights, or just a few hours instead.

7. creation_date. Upon creation every object that results from processing has an attribute that stores the moment of its creation. This information is relevant as "Newer is better" is a general rule for objects to determine which calibration data item should be applied to them from the pool of applicable calibration data items.

Handling of KiDS survey data starts by filtering the pool of available data on which the survey handling should act. This is called setting a 'Context' in Astro-WISE, and is done using the parameters 'Project' and 'Privileges' described above. After logging into Astro-WISE, the KiDS team member selects the Project 'KIDS' and a minimum Privileges level of 1 or 2 . All results the member produces will be part of the 'KIDS' Project, and the member is able to see all data available within 'KIDS'.

The baseline KiDS survey products are all part of the Project 'KIDS' and reside at privileges level 2 (named PROJECT). These data can be accessed only by KiDS survey team members. Each KiDS team member can experiment in her/his own privileges level 1 (MYDB) to create improved versions of these baseline products. Only the single team member can access survey data at MYDB level and promote it to the PROJECT level. A KiDS project manager can promote baseline survey data from privileges level 2 to all higher levels. Survey data at privileges level 3 (ASTRO-WISE) can be accessed by all Astro-WISE users. At privileges level 4 (WORLD) the data become publicly accessible, that is to users without an Astro-WISE account (anonymous users). Finally at priveleges level 5 (VO), the data are accessible also from the Virtual Observatory.

Thus, it is the combination of Context parameters user, Project and Privileges level that determine what data is filtered to be accessible for survey 
handling. Data handling itself is done either by using a command-line interface (CLI) or via webservices. How-to's and tutorials for using the CLI can be found online, ${ }^{5}$ and for a description of available webservices we refer to the paper on Astro-WISE interfaces in this issue [3].

\subsection{Quality assessment and control}

Three types of quality assessment are facilitated in Astro-WISE:

1. "verify": automatic verification by the system upon creation of a data object

2. "compare": comparison of a processing result to an earlier similar result

3. "inspect": manual inspection by a human user of a data object

Although this approach is generic, and all classes of data objects resulting from processing (i.e., "processing targets") have the three types of methods implemented, the actual content of the method is specific for the type of data. The result of the application of a method is stored as a data attribute, namely either the quality_flags or the is_valid attribute discussed in the previous section. Additional quality control information can be stored in a Comment object as a free string that links to the process target. The quality of an end product depends on the pipeline configuration and the quality of (calibration) data at all intermediate processing stages. This generic approach to quality assessment together with the data lineage in Astro-WISE (for details see the paper on data lineage in this issue [24]) facilitates tracing back these dependencies.

The QualityWISE webservice provides an overview of the quality assessment information (verdicts, inspection figures, numbers) for human inspection, including links to the QualityWISE pages of data items on which it depends. Figure 4 gives an impression of this service and for an in-depth discussion we refer to the QualityWISE paper in this issue [20]. To zoom in on quality issues, the user can use the webservice for database querying and viewing (DBViewer ${ }^{6}$ ) which provides links to the QualityWISE page of returned data items. At the command-line the user can customize the quality assessment to particular needs with maximum freedom. For example, batch scripts can be used for mass quality control, with switches to an interactive mode when needed.

For quality control it is important that baseline data products readily distinguished from experimental versions of data products. The Privileges levels discussed earlier serve to keep such versions apart. A KiDS team member can experiment to improve data quality at the MYDB level. At this level, project data at all Privileges levels is available, but the resulting products are only visible to the team member. Bad outcomes are discarded by invalidating the

\footnotetext{
${ }^{5}$ http://www.astro-wise.org/portal/aw_howtos.shtml

${ }^{6} \mathrm{http} / / / \mathrm{dbview}$.astro-wise.org
} 


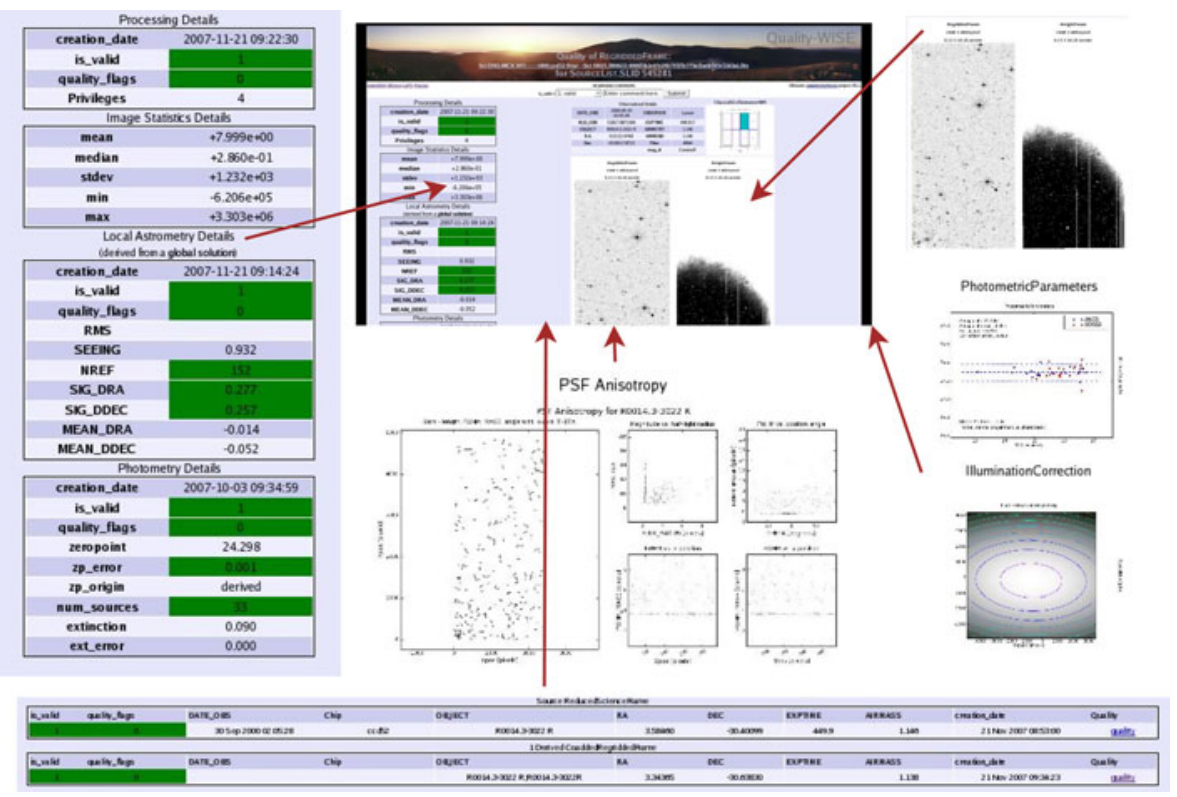

Fig. 4 The QualityWISE webservice bundles quality information for a single data object. An example page is shown partially, with arrows linking to zoom ins of the different sections of the webpage. See Quality Control paper in this issue [20] for full discussion of this webservice and quality control in Astro-WISE in general

data. Promising outcomes can be shared with the team by publishing to the PROJECT level (see Fig. 5). Fellow team members can then inspect the data and provide feedback (e.g., using Comments objects in Astro-WISE). The final verdict is set in the is_valid attribute $(0=\mathrm{bad}, 2=$ good $)$. Upon team acceptance the survey object becomes baseline and can be published higher up, eventually for delivery to the outside world. Compromises in data quality identified only after publishing can be handled adequately. For example, owing to the data lineage provided by Astro-WISE, database queries with few lines of code can isolate all data derived using a specific calibration file. These can then easily be invalidated at all privileges levels.

\subsection{Survey calibration control}

Like science data, calibration data are represented as objects in Astro-WISE. In addition to quality parameters, these objects carry a creation date and editable timestamps that mark their validity period. A request for a processing target generates a database query that returns all good-quality calibration objects with a validity period that covers the observation date. The newest good-quality calibration object is then selected, following the survey handling rule "newer is better". 


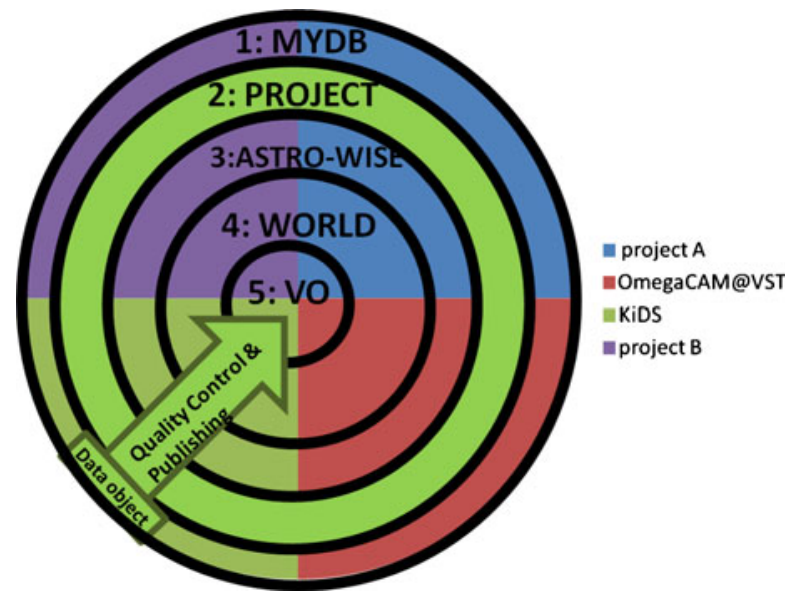

Fig. 5 Operational levels at which survey handling acts. This is configured by setting the minimum Privileges level in the user's Astro-WISE context. Each annulus represents a Privileges level. The operational level includes the annulus and all levels interior to it. Each color represents a project (KiDS in denoted in green). The baseline KiDS survey products reside at 2:PROJECT. These data can be accessed only by KiDS survey team members. Each team member can experiment to create improved versions of baseline products in her/his own level 1:MYDB, where data is only accessible by the single team member. If content, the member promotes the products to 2:PROJECT to share them with the team. The KiDS project manager can publish baseline survey data from 2 to levels 3-5. Survey data at 3:ASTRO-WISE can be accessed by all Astro-WISE users. At 4:WORLD, the data become accessible additionally to the astronomical community without an Astro-WISE account (anonymous users). At 5: $\mathrm{VO}$, the data are accessible also from the Virtual Observatory

The calibration scientists in the KiDS team, who are spread over Europe, collaborate using the calibration control webservice $\mathrm{CalTS}^{7}$ to manipulate this eclipsing of older calibrations by new ones (see Fig. 6 and paper on user interfaces in this issue [3] for details). As for science data, the Context parameters are used to limit the survey calibration operations to the appropriate subset of calibration data available in the system. As the survey progresses the calibration scientists will build up a set of calibration objects with a continuous time coverage. This build up of calibration data will make subtle trends as a function of observational state (instrument configuration, telescope position, atmospheric state) statistically significant. Investigation of such trends can be done using the Astro-WISE CLI in combination with scripts that can be as short as a few lines of code. The resulting deeper physical understanding of the OmegaCAM instrument and the Paranal atmosphere will lead to better calibrations which eclipse the older ones. The final result is that the instrument plus atmosphere become continuously calibrated rather than establishing calibrations on a per dataset basis. This continuous calibration

\footnotetext{
${ }^{7} \mathrm{http} / / /$ calts.astro-wise.org/
} 


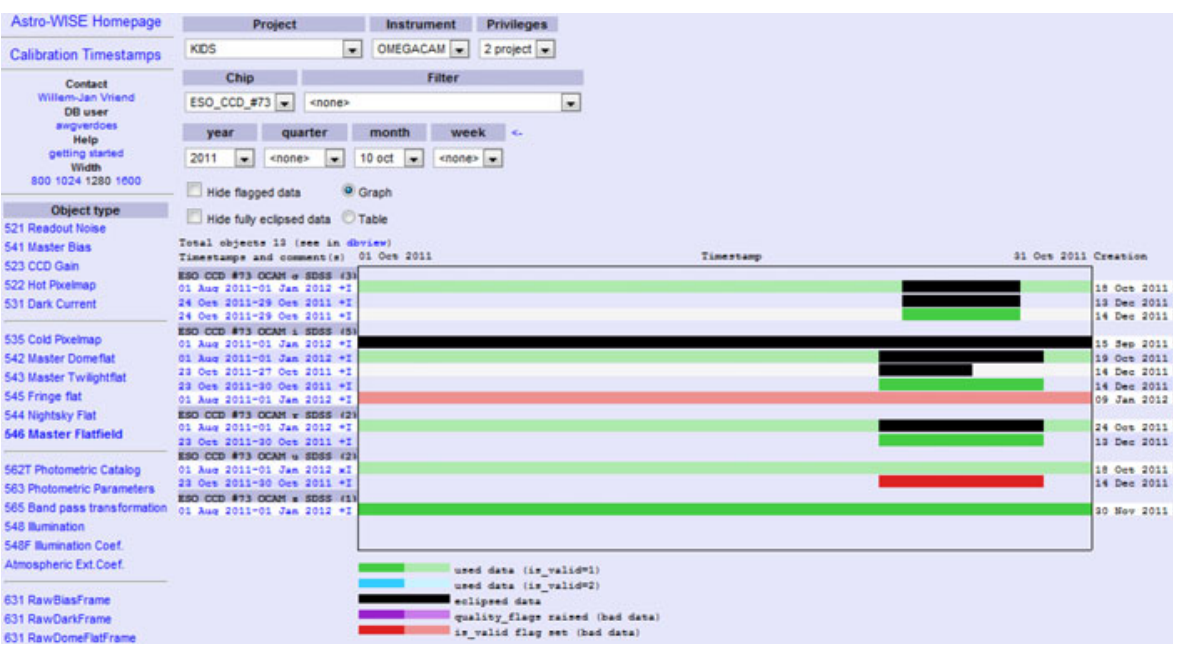

Fig. 6 Snapshot of the Calibration Time Stamp editor webservice (CalTS). All types of calibration data can be selected and their information graphically depicted. Horizontal bars show the time range validity of an object. The vertical stacking of the bars is ordered by creation date, with newer ones (green) eclipsing older versions (black). Other color codings depict the quality assessment verdicts. The validity time ranges and quality assessment parameters can be adjusted. See paper on User Interfaces in this issue [3] for technical details

coverage can be pooled with all Astro-WISE users working on OmegaCAM data by publishing the data to privileges 3 or higher.

\section{From first reductions towards final data delivery}

KiDS survey operations have started officially on 15 October 2011. The KiDS survey fields are currently being calibrated with initial calibration data. Quality control on these calibration data and the processed science data will lead to improvements of both the calibration data objects as well as of the pipeline configuration and methods. This in turn will allow the production of improved versions of the initial survey data products. Owing to the direct access to data lineage in Astro-WISE it is straightforward to re-process only the data objects that are affected by these changes. The resulting high quality, calibrated survey data can then be used for the production of advanced products, such as photometric redshifts, galaxy morphometry, source variability analysis.

Following this approach the KiDS team will move from a 'quick-look' version of the first survey products towards publishing a complete, high quality, value-added KiDS Public Survey data set. The synergy with the different techniques and approaches used within the team, including independent data processing routes (e.g. THELI [9] and VSTtube [11] will allow for constant cross-checking and improvements. All the time, the team will benefit from 
Astro-WISE as its 'live archive', that captures the accumulation of knowledge about OmegaCAM, VST and the KiDS survey data over the years.

Acknowledgements This work is financially supported by the Netherlands Research School for Astronomy (NOVA) and Target. ${ }^{8}$ Target is supported by Samenwerkingsverband Noord Nederland, European fund for regional development, Dutch Ministry of economic affairs, Pieken in de Delta, Provinces of Groningen and Drenthe. Target operates under the auspices of Sensor Universe. The authors thank the referee for the constructive comments that helped to improve the paper.

Open Access This article is distributed under the terms of the Creative Commons Attribution License which permits any use, distribution, and reproduction in any medium, provided the original author(s) and the source are credited.

\section{References}

1. Abazajian, K.N., et al.: The seventh data release of the Sloan Digital Sky Survey. ApJS 182, 543 (2009)

2. Begeman, K.G., Belikov, A.N., Boxhoorn, D., Valentijn, E.: WISE technologies. Exp. Astron. (Special Issue: Astro-WISE) (2012, in preparation)

3. Belikov, A.N., Vriend, W.-J., Sikkema, G.: Astro-WISE interfaces-scientific information system brought to the user. Exp. Astron. (Special Issue: Astro-WISE) (2012, in preparation)

4. Belokurov, V., et al.: The field of streams: Sagittarius and its siblings. ApJ 642, L137 (2006)

5. Belokurov, V., et al.: Cats and dogs, hair and a hero: a quintet of new Milky Way companions. ApJ 654, 897 (2007)

6. Colless, M., et al.: The 2dF Galaxy Redshift Survey: spectra and redshifts. MNRAS 328, 1039 (2001)

7. Cooper, M.C., Griffith, R.L., Newman, J.A., et al.: The DEEP3 Galaxy Redshift Survey: the impact of environment on the size evolution of massive early-type galaxies at intermediate redshift. MNRAS 419, 3018 (2012)

8. Drinkwater, M.J., Jurek, R.J., Blake, C., et al.: The WiggleZ Dark Energy Survey: survey design and first data release. MNRAS 401, 1429 (2010)

9. Erben, T., Hildebrandt, H., Lerchster, M., et al.: CARS: the CFHTLS-Archive-Research Survey. I. Five-band multi-colour data from 37 sq. deg. CFHTLS-wide observations. A\&A 493, 1197 (2009)

10. Franx, M., Illingworth, G., Heckman, T.: Multicolor surface photometry of 17 ellipticals. AJ 98, 538 (1989)

11. Grado, A., Capaccioli, M., Limatola, L., Getman, F.: VST processing facility: first astronomical applications. Memorie della Societa Astronomica Italiana Supplement 19, 362 (2012)

12. Harris, H.C., Gates, E., Gyuk, G., et al.: Additional ultracool white dwarfs found in the Sloan Digital Sky Survey. ApJ 679, 697 (2008)

13. Jain, B., Zhang, P.: Observational tests of modified gravity. PhRvD 78, 063503 (2008)

14. Kilic, M., Munn, J.A., Williams, K.A., et al.: Visitors from the Halo: 11 Gyr old white dwarfs in the solar neighborhood. ApJ 715, L21 (2010)

15. Kuijken, K.: OmegaCAM: ESO's newest imager. ESO Messenger 146, 8 (2011)

16. Kuijken, K., Rich, R.M.: Hubble Space Telescope WFPC2 proper motions in two bulge fields: kinematics and stellar population of the galactic bulge. AJ 124, 2054 (2002)

17. Lotz, J.M., Jonsson, P., Cox, T.J., et al.: The major and minor galaxy merger rates at $\mathrm{z}<1.5$. ApJ 742, 103 (2011)

18. Man, A.W.S., Toft, S., Zirm, A.W., Wuyts, S., van der Wel, A.: The pair fraction of massive galaxies at $0 \leq \mathrm{z} \leq 3$. ApJ 744, 85 (2012)

\footnotetext{
${ }^{8}$ www.rug.nl/target
} 
19. Mandelbaum, R., et al.: Density profiles of galaxy groups and clusters from SDSS galaxygalaxy weak lensing. MNRAS 372, 758 (2006)

20. McFarland, J.P., Helmich, E.M., Valentijn, E.A.: The Astro-WISE approach to quality control for astronomical data. Exp. Astron. 129, arXiv:1203.4208 (2012, submitted)

21. McFarland, J.P., Verdoes Kleijn, G., Sikkema, G., et al.: The Astro-WISE optical image pipeline: development and implementation. Exp. Astron. 129, arXiv:1110.2509 (2012, accepted)

22. Mellier, Y.: Probing the Universe with weak lensing. ARA\&A 37, 127 (1999)

23. Miller, L., Kitching, T.D., Heymans, C., Heavens, A.F., van Waerbeke, L.: Bayesian galaxy shape measurement for weak lensing surveys - I. Methodology and a fast-fitting algorithm. MNRAS 382, 315 (2007)

24. Mwebaze, J., Boxhoorn, D., Valentijn, E.A.: Data lineage in scientific data processing. Exp. Astron. (Special Issue: Astro-WISE) (2012, in preparation)

25. Parker, L.C., Hoekstra, H., Hudson, M.J., van Waerbeke, L., Mellier, Y.: The masses and shapes of dark matter halos from galaxy-galaxy lensing in the CFHT Legacy Survey. ApJ 669, 21 (2007)

26. Peacock, J.A., et al.: ESA-ESO working group on fundamental cosmology. ESO/ESA Working Group 3 Report (2006)

27. Peng, C.Y., Ho, L., Impey, C.D., Rix, H.-W.: Detailed structural decomposition of galaxy images. AJ 124, 266 (2002)

28. Schlegel, D., White, M., Eisenstein, D.: Astro2010: the astronomy and astrophysics decadal survey. Science White Papers, no. 314 (2009)

29. Skrutskie, M.F., Cutri, R.M., Stiening, R., et al.: The Two Micron All Sky Survey (2MASS). AJ 131, 1163 (2006)

30. Spergel, D.N., et al.: Three-year Wilkinson Microwave Anisotropy Probe (WMAP) observations: implications for cosmology. ApJS 170, 377 (2007)

31. Valentijn, E.A., et al.: Astro-WISE: chaining to the Universe. ASPC 376, 491 (2007)

32. van Daalen, M.P., Schaye, J., Booth, C.M., Dalla Vecchia, C.: The effects of galaxy formation on the matter power spectrum: a challenge for precision cosmology. MNRAS 415, 3649 (2011)

33. Verdoes Kleijn, G., Valentijn, E., Kuijken, K., et al.: OmegaCAM plus Astro-WISE: an InfoScope. Exp. Astron. 129 (2012, in preparation)

34. Verdoes Kleijn, G., Belikov, A.N., Heraudeau, P., et al.: The Data Zoo in Astro-WISE. Exp. Astron. (Special Issue: Astro-WISE) (2012, submitted)

35. Zhao, G.-B., Zhang, X.: Probing dark energy dynamics from current and future cosmological observations. PhRvD 81, 043518 (2010) 